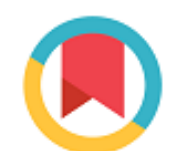

Check for updates

\title{
Superselective Angioembolisation: A Report of Two Rare Cases of Hemoptysis
}

Mohammad Sadegh Keshmiri ${ }^{1}$, Babak Sharifkashani ${ }^{2}$, Alireza Serati ${ }^{1,}$, , Seyed Reza Seyedi ${ }^{1}$, Farah Naghashzadeh ${ }^{3}$, Siroos Salehi ${ }^{1}$, Omid Dehghan ${ }^{1}$

${ }^{1}$ Masih Deveshvari Hospital, Shahid Beheshti University of Medical Sciences, Tehran, Iran

${ }^{2}$ Tobacco Prevention and Control Research Center (TPCRC), National

Research Institute of Tuberculosis and Lung Disease (NRILTD), Shahid

Beheshti University of Medical Sciences, Tehran, Iran

${ }^{3}$ Lung transplantation Research Center, National Research Institute of Tuberculosis and Lung Disease(NRILTD), Shahid Beheshti University of Medical Sciences, Tehran, Iran

${ }^{*}$ Corresponding author: Ali Reza Serati, Masih Deveshvari Hospital, Shahid Beheshti University of Medical Sciences, Tehran, Iran, E-mail:

DOI: $10.21859 / \mathrm{ijcp}-402$ Saratiali@yahoo.com

\begin{tabular}{l}
\hline Submitted: $18-12-2018$ \\
Accepted: 22-01-2018 \\
\hline Keywords: \\
Hemoptysis \\
Embolization, Therapeutic \\
Bronchiectasis \\
\hline C 2019. International Journal of \\
Cardiovascular Practice.
\end{tabular}

\begin{abstract} Superselective bronchial embolization is recently performed with a high success rate. The current study aimed at discussing the procedure and reporting two cases underwent embolization in the Masih-Daneshvari Hospital, a large center for this procedure, in Iran.
\end{abstract}

Cardiovascular Practice.

\section{INTRODUCTION}

Bronchial arteries embolization (BAE) is mainly performed in moderate to severe hemoptysis cases irresponsive to medical managements [1]. This procedure remains a palliative procedure for management of hemoptysis in patients who are not candidate for more invasive treatments such as surgery. Most common reported underlying diseases are tuberculosis (TB), bronchiectasis, and aspergillomas [1]. Short-term success and immediate bleeding cessation were high; however, recurrence may occur. Recurrence may be due to incomplete embolization, recanalization of previously embolized vessels, and recruitment of new collaterals due to main disease progression. Presence of non-bronchial systemic collaterals, abnormal site of collaterals (e g, coronary arteries), bronchopulmonary shunting, aspergillomas reactivation, and multidrug resistant $\mathrm{TB}$ are associated with higher recurrence rates. After 2010, most published reports emphasized superselective embolization to decrease procedural complications and administration of polyvinyl alcohol (PVA) in lower doses [1].

The initial control rate of hemoptysis was reported $96 \%$ (21 of 22 ) in a study on a superselective group and $88 \%$ (22 of 25) in the non-superselective group. Cumulative hemoptysis control rates of the super-selective and nonsuperselective groups were $80 \%$ and $67 \%$ at six months, $79 \%$ and $56 \%$ at one and two years, and $79 \%$ and $48 \%$ at three years, respectively; although the difference was insignificant using the generalized Wilcoxon test. One major complication (spinal infarction) occurred in the non-superselective group [2]. Superselective embolization enabled bypassing spinal arteries and abolished smaller and more distal and torturous arteries, which led to better control of hemoptysis and lower risk of complications.

\section{CASE PRESENTATION}

Here is the report of two successful cases of rare superselective BAE. The first case was a 34-year-old male with the history of bronchiectasis presented with massive hemoptysis. Based on chest computed tomography (CT) scan images, bleeding source was in the right side. The patient was scheduled for the embolization of bronchial arteries originated from thoracic region. The procedure was performed with PVA successfully with no complications. Seven days later, the patient returned and this time CT scan images showed the left sided bleeding. 


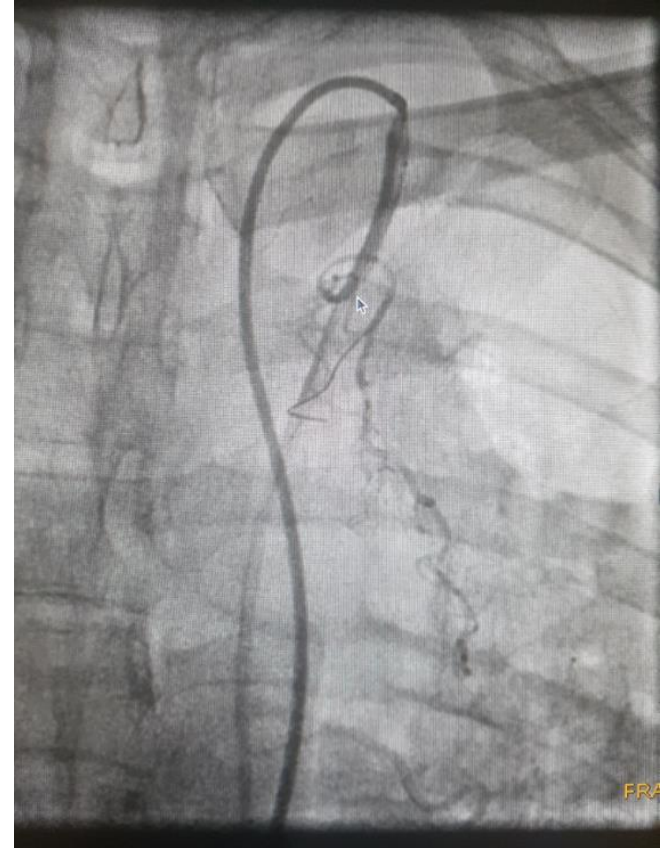

Figure 1. Superselective embolization in a branch artery originating from left internal mammary artery

Then the patient underwent angiography and the target vessels were detected as some branches originating from left internal mammary artery (Fig 1). Selective embolization was performed on the determined vessels. For this propose, a $6 \mathrm{~F}$ guide catheter was inserted. A 0.014-inch hydrophilic guidewire (pilot 50 Abbott Vascular, Ltd.) was passed through the tortuous vessel with a 2.7-Fr. microcatheter (Rebar 18, Ev3 Co.).

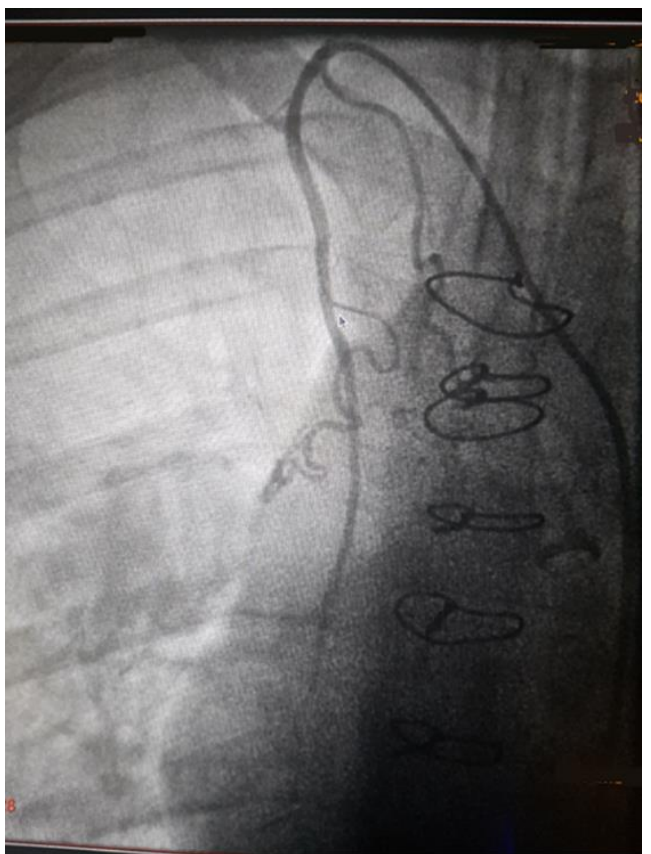

Figure 2. Superselective embolization in a branch artery originating from right internal mammary artery

Two milliliters of PVA 700 was injected via the microcatheter into the target vessels. The patient was admitted to the intensive care unit and discharged after two days with no hemoptysis. Three months follow-up showed no sign of bleeding recurrence.

In the second case, a 32-year-old male patient with chronic thromboembolic pulmonary hypertension, aberrant bronchial vessel originated from right internal mammary artery (Fig 2). After embolizing the thoracic branches, superselective embolization was performed on the aberrant vessel using the aforementioned technique. Both procedures were performed with the injection of 3000 units of intravenous heparin, BiPAP oxygen mask to perform $\mathrm{O}_{2}$ saturation $>90 \%$, and 6-Fr right transfemoral artery sheath size.

\section{CONCLUSIONS}

In patients with hemoptysis, BAE can be a curative procedure after medical treatment or may be a bridge therapy before surgery for stabilizing the patient's conditions and postponing the surgery until the stabilization of patient's conditions. Although surgery is a definitive therapy, it carries a mortality of $18 \%$ when performed electively, which can increase to $40 \%$ when performed emergently [3]. Bronchial embolization is a minimally invasive procedure, which can be performed in critically ill patients.

Angiographically, the bronchial arteries arise from the descending thoracic aorta between the upper $\mathrm{t} 5$ and the lower t6 vertebral bodies in $70 \%$ of the normal population. Another $10 \%$ remain a first order branch of the thoracic aorta or arch, but outside of the $\mathrm{t5}-\mathrm{t} 6$. The remaining $20 \%$ originate from a variety of structures including the thoracic (brachiocephalic, subclavian, internal mammary, pericardiopherenic, or thyrocervical) and abdominal (aorta, inferior phrenic or celiac) branches [4-6].

Non-bronchia collaterals should be assessed and treated concurrently with the bronchial arteries at the time of initial arteriogram. In the two reported cases, two nonbronchial collaterals were successfully embolized following the embolization of bronchial arteries. Likewise, the application of microcatheter in a coaxial technique is now well documented for selective catheterization and administration of embolic agents $[7$, 8]. Technical success rate is reported $90 \%$ [9].

Amongst articles published before 2010, superselective embolization was notably reported in four studies [10]. In the current study center, the success rate of bronchial embolization was about $92 \%$ and complication rate $10 \%$. Right bronchial arteries are the most common bronchials embolized in previous reports, while nonbronchial systemic collaterals were embolized in the first session in about $40 \%-62 \%$ of patients in different reports [11].

The intercostal and internal mammary arteries were the most frequently embolized non-bronchial systemic collaterals [11].

Ramakantan et al., recommended not to embolize nonbronchial systemic collaterals in the first session [12]. 
Although it is a safe procedure, the search for nonbronchial arteries reduces the recurrence rate [13]. In the current study center, the non-bronchial arteries were routinely examined in the same session, which helped us with the better control of hemoptysis. For the non-bronchial arteries, superselective embolization is preferred due to lower complications and better outcomes. In other bronchials, superselective embolization did not perform routinely and our findings were similar to those of other studies. The nonsuperselective embolization is easy to perform and costeffective. However, the shorter duration and less contrast injection are the other advantages of the procedure.

Neurologic complications due to spinal cord ischemia leading to transient or permanent paraparesis or paraplegia occur in $0.6 \%-4.4 \%$ of the cases [14-16]. It is mostly attributed to inadvertent embolization of spinal arteries arising from bronchial or intercostobronchial arteries. However, in two studies with the incidence of paraparesis, spinal arteries were not visualized even on retrospective review of the digital subtraction angiography images. Although some authors considered visualization of anterior spinal artery as a contraindication for BAE [14-16], superselective catheterization enabled safer embolization in most studies.

\section{REFERENCE}

1. Panda A, Bhalla AS, Goyal A. Bronchial artery embolization in hemoptysis: a systematic review. Diagn Interv Radiol. 2017;23(4):307-17. doi: 10.5152/dir.2017.16454 pmid: 28703105

2. Tanaka N, Yamakado K, Murashima S, Takeda K, Matsumura $\mathrm{K}$, Nakagawa $\mathrm{T}$, et al. Superselective bronchial artery embolization for hemoptysis with a coaxial microcatheter system. J Vasc Interv Radiol. 1997;8(1 Pt 1):65-70. doi: 10.1016/S1051-0443(97)70517-7 pmid: 9025041

3. Fernando HC, Stein M, Benfield JR, Link DP. Role of bronchial artery embolization in the management of hemoptysis. Arch Surg. 1998;133(8):862-6. doi: 10.1001/archsurg.133.8.862 pmid: 9711960
4. Marshall TJ, Jackson JE. Vascular intervention in the thorax: bronchial artery embolization for haemoptysis. Eur Radiol. 1997;7(8):1221-7. doi: 10.1007/s003300050279 pmid: 9377505

5. Stoll JF, Bettmann MA. Bronchial artery embolization to control hemoptysis: a review. Cardiovasc Intervent Radiol. 1988;11(5):263-9. doi: 10.1007/BF02577032 pmid: 3145138

6. Botenga SJ. Selective bronchial and intercostal arteriography. Baltimore: Williams and wilkins; 1970.

7. Najarian KE, Morris CS. Arterial embolization in the chest. J Thorac Imaging. 1998;13(2):93-104. doi: 10.1097/00005382-199804000-00004 pmid: 9556286

8. Jiang S, Sun XW, Yu D, Jie B. Endovascular embolization of bronchial artery originating from the upper portion of aortic arch in patients with massive hemoptysis. Cardiovasc Intervent Radiol. 2014;37(1):94-100. doi: 10.1007/s00270-013-0638-7 pmid: 23674273

9. Ramakantan R, Bandekar VG, Gandhi MS, Aulakh BG, Deshmukh HL. Massive hemoptysis due to pulmonary tuberculosis: control with bronchial artery embolization. Radiology. 1996;200(3):691-4. doi: 10.1148/radiology.200.3.8756916 pmid: 8756916

10. Yu-Tang Goh P, Lin M, Teo N, En Shen Wong D. Embolization for hemoptysis: a six -year review. Cardiovasc Intervent Radiol. 2002;25(1):17-25. doi: 10.1007/s00270001-0047-1 pmid: 11907769

11. White RI, Jr. Bronchial artery embolotherapy for control of acute hemoptysis: analysis of outcome. Chest. 1999;115(4):912-5. pmid: 10208183

12. Sopko DR, Smith TP. Bronchial artery embolization for hemoptysis. Semin Intervent Radiol. 2011;28(1):48-62. doi: 10.1055/s-0031-1273940 pmid: 22379276

13. Anuradha C, Shyamkumar NK, Vinu M, Babu NR, Christopher DJ. Outcomes of bronchial artery embolization for life-threatening hemoptysis due to tuberculosis and posttuberculosis sequelae. Diagn Interv Radiol. 2012;18(1):96101. doi: 10.4261/1305-3825.DIR.3876-11.2 pmid: 21678246

14. Agmy GM, Wafy SM, Mohamed SA, Gad YA, Mustafa H, Abd El-Aziz AE-S. Bronchial and nonbronchial systemic artery embolization in management of hemoptysis: experience with 348 patients. ISRN Vasc Med. 2013;2013(26):254-9.

15. Fruchter O, Schneer S, Rusanov V, Belenky A, Kramer MR. Bronchial artery embolization for massive hemoptysis: longterm follow-up. Asian Cardiovasc Thorac Ann. 2015;23(1):55-60. doi: 10.1177/0218492314544310 pmid: 25053662

16. Remy J, Arnaud A, Fardou H, Giraud R, Voisin C. Treatment of hemoptysis by embolization of bronchial arteries. Radiology. 1977;122(1):33-7. doi: 10.1148/122.1.33 pmid: 830351 